\title{
Wie Kommunen systematisch ihre Risiken managen
}

\author{
Michael Gerstacker/Stephanie Blättler
}

Risikomanagement gewinnt für Kommunen zunehmend an Bedeutung. Häufig bestehen jedoch nur vereinzelte und eher vage Vorstellungen darüber, wie sicher (bzw. risikobehaftet) Prognosen, Planwerte und Prozesse sind. Um herauszufinden, weshalb Risikomanagement in Kommunen mit unterschiedlicher Priorität betrieben wird, welche Hindernisse sich bei der Einführung und „im laufenden Betrieb“ ergeben und welche Rahmenbedingungen ein erfolgreiches Risikomanagement begünstigen, hat die KGSt unter Begleitung der Hochschule Luzern eine Umfrage durchgeführt. Danach hält eine Mehrheit der Befragten Risikomanagement zur Verbesserung der Verwaltungssteuerung für unverzichtbar. Zeitmangel, begrenzte Personalressourcen, fehlendes (Fach-)Wissen der Beschäftigten und eine unzureichende Unterstützung durch die kommunalen Entscheidungsträger stehen einer Einführung jedoch oftmals entgegen. Die Umfrageergebnisse bieten Gelegenheit, die örtliche Situation zu reflektieren, seitens der Verantwortlichen die Initiative $z u$ ergreifen und Impulse für ein systematisches Risikomanagement zu setzen.

In der Privatwirtschaft wird Risikomanagement häufig als zwingende Notwendigkeit angesehen. ${ }^{1}$ Auch für Kommunen ist die Auseinandersetzung mit Risiken nicht neu: Auf unterschiedlichen (Hierarchie-)Ebenen werden politische Mandatsträger, Führungskräfte und Beschäftigte mit unsicheren Ereignissen konfrontiert. Sie müssen auf alternative Entwicklungen vorbereitet sein und mit Risiken verantwortungsbewusst umgehen. Dennoch bereitet es vielen Kommunen Schwierigkeiten, Risiken (und Chancen) systematisch $\mathrm{zu}$ identifizieren, $\mathrm{zu}$ bewerten und zu steu-

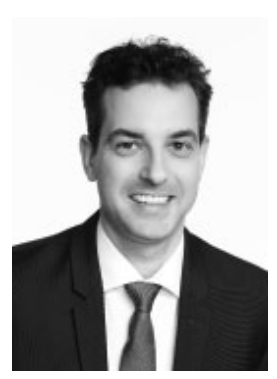

Michael Gerstacker

Dipl.-Kfm., Referent im Programmbereich Finanzmanagement der KGSt ern. Risikobetrachtungen erfolgen oft nur intuitiv und unterliegen keiner systematischen Vorgehensweise. Häufig besteht nur eine vage Vorstellung darüber, wie sicher (bzw. risikobehaftet) Prognosen, Planwerte und Prozesse sind.

Risiken sind alle Ereignisse innerhalb und außerhalb von Verwaltungen, die sich ungünstig auf die kommunale Zielerreichung auswirken können. ${ }^{2}$ Aufgabe des Risikomanagements ist es, potenzielle Abweichungen von zuvor definierten Zielen frühzeitig zu erkennen und bei Bedarf ent-

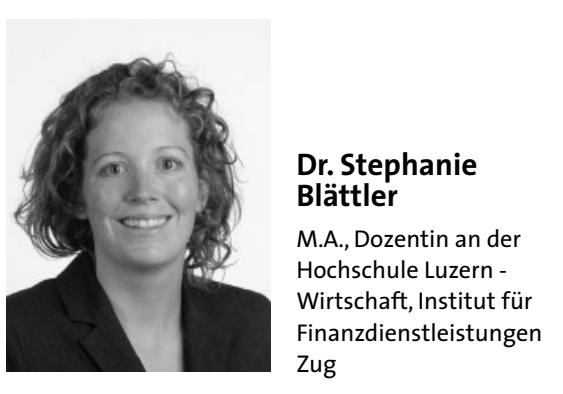

sprechende Gegensteuerungsmaßnahmen einzuleiten. ${ }^{3}$ Im Idealfall stellt es darüber hinaus sicher, dass neben Risiken auch Chancen erkannt, bewertet und bei der Entscheidungsfindung berücksichtigt werden. ${ }^{4}$

Um einen Überblick über das Risikomanagement in deutschen Kommunen zu gewinnen, hat die KGSt mit wissenschaftlicher Begleitung der Hochschule Luzern eine standardisierte Online-Befragung unter ihren Mitgliedern durchgeführt. ${ }^{5}$ Die Umfrage liefert wichtige Erkenntnisse über den Umsetzungsstand und die Rahmenbedingungen, die für ein erfolgreiches Risikomanagement in Kommunen zweckdienlich sind.

122 Umfrageteilnehmer/-innen haben den Fragebogen vollständig ausgefüllt und die Umfrage abgeschlossen (Bearbeitungsdauer des Fragebogens: 10-15 Minuten). ${ }^{6}$ Die Antworten stammen zu 65 Prozent von Beschäftigten aus den Bereichen Finanzen/Kämmerei, Controlling/ Risikomanagement und Rechnungsprüfung. Beteiligt haben sich auch Akteure aus Verwaltungsleitung und Politik sowie Beschäftigte aus unterschiedlichen (Fach-) Bereichen und Ämtern. Rund 70 Prozent

1 Ein systematischer und dokumentierter Umgang mit Risiken ist beispielsweise durch das Gesetz zur Kontrolle und Transparenz im Unternehmensbereich (KonTraG) gesetzlich vorgeschrieben.

2 Vgl. KGSt 2011, S. 23.

3 Vgl. z.B. Blättler/Hunziker 2015, S. 83.

4 Vgl. Budäus/Hilgers 2009, S. 22.

5 Vgl. KGSt 2019.

6 Es erfolgten zwei Aufrufe im KGSt ${ }^{\circ}$-Journal und die gezielte Ansprache von weiteren 1.040 Mailadressen der KGSt. Fragebögen mit unvollständigen Antwortangaben wurden bei der Auswertung ausgeschlossen. 


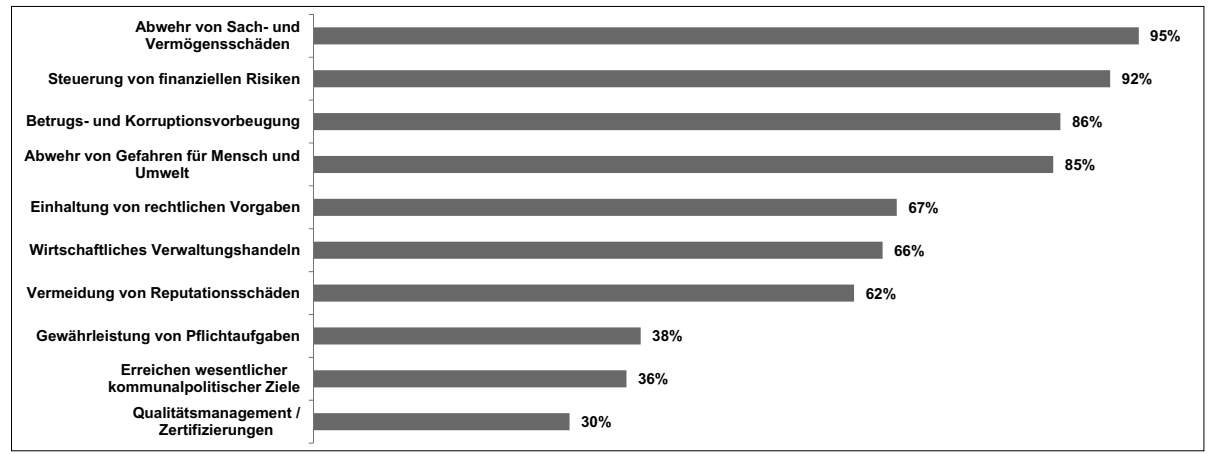

Abb. 1: Zweck eines strukturierten und dokumentierten Risikomanagements ( $n=$ jeweils 122)

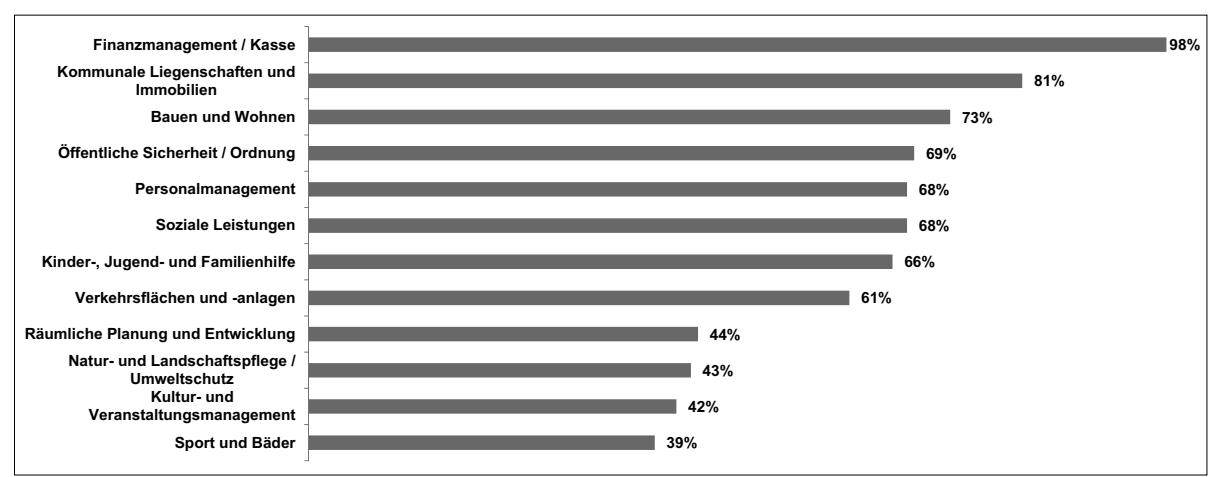

Abb. 2: Mögliche (Teil-)Bereiche für ein strukturiertes und dokumentiertes Risikomanagement $(n=$ jeweils 122)

der Umfrageteilnehmer/-innen gaben an, in leitender Funktion tätig zu sein.

\section{Zweck und Einsatzbereiche eines kommunalen Risikomanagements}

Nach Ansicht der Umfrageteilnehmer/innen besteht der Zweck eines kommunalen Risikomanagements vor allem darin, Sach- und Vermögensschäden abzuwehren, finanzielle Risiken zu steuern, Betrug und Korruption vorzubeugen sowie Gefahren für Mensch und Umwelt abzuwehren (vgl. Abb. 1).

Da die Umfrageteilnehmer/-innen der Steuerung von finanziellen Risiken einen hohen Stellenwert beimessen, ist es naheliegend, dass ein systematisches Risikomanagement insbesondere im Finanzbereich für erforderlich gehalten wird (vgl. Abb. 2). Eine Mehrheit der Befragten hält Risikomanagement aber auch in den Bereichen kommunale Liegenschaften und Immobilien, Bauen und Wohnen, Öffentliche Sicherheit und Ordnung, Personalmanagement, Soziale Leistungen, Verkehrsflächen und -anlagen sowie in der Kinder-, Jugend und Familienhilfe für notwendig.

Dies ergibt sich z.T. bereits aus spezialgesetzlichen Vorschriften, die einen strukturierten Umgang mit Risiken erfordern.
So finden sich beispielsweise in $\mathbb{\int}$ a SGB VIII konkrete Vorgaben zum Schutzauftrag bei Kindeswohlgefährdung und zur Abschätzung des Gefährdungsrisikos. Weitere Vorschriften, die einen strukturierten Umgang mit Risiken erfordern, enthalten z.B. die Richtlinien zur Betreiberverantwortung bei kommunalen Gebäuden, das Arbeitsschutzgesetz in Bezug auf die Verhütung von Unfällen bei der Arbeit oder die Verwaltungsvorschriften zum Brandenburgischen Brand- und Katastrophenschutzgesetz.

Allerdings halten nur 36 Prozent der Umfrageteilnehmer/-innen Risikomanagement zur Erreichung von wesentlichen kommunalpolitischen Zielen für erforderlich. Dieser Befund ist bemerkenswert, da Risiken sich ungünstig auf die kommunale Zielerreichung auswirken können. Ziele und Risiken sind zwei Seiten derselben Medaille.

Die Umfrageergebnisse verdeutlichen andererseits die von der Privatwirtschaft abweichende Zwecksetzung des kommunalen Risikomanagements: Während Risikomanagement im privaten Sektor vornehmlich zur Erreichung wirtschaftlicher Ziele und zur Steuerung von finanziellen Risiken dient, ist es Aufgabe von Kommunen, allgemeinwohlorientiert $\mathrm{zu}$ handeln, den gesetzlichen Verpflichtungen nachzukommen und die öffentlichen Mittel so einzusetzen, dass eine stetige Aufgabenerfüllung gewährleistet ist. ${ }^{7}$ Neben der finanziellen Leistungsfähigkeit ist der Schutz der Bevölkerung sicherzustellen. ${ }^{8}$ Die alleinige Betrachtung von finanziellen Risiken reicht für Kommunen nicht aus.

Gleichwohl sind vor allem die Finanzverantwortlichen in Kommunen wesentliche Treiber für einen systematischen Umgang mit Risiken. Der Umstand, dass nahezu jeder Risikoeintritt mittelbar Auswirkungen auf den Haushalt hat, ist für Finanzverantwortliche möglicherweise Anlass, ein systematisches Risikomanagement zu fordern. Die Einführung eines Tax-Compliance-Management-Systems oder die Umsetzung der GoBD ${ }^{9}$ sind weitere potenzielle Gründe, Risikomanagement aktiv voranzutreiben.

In der täglichen Steuerung ist Risikomanagement hingegen Aufgabe der gesamten operativen Ebene, also aller Fachbereiche, -ämter und -abteilungen. ${ }^{10}$ Nur sie kennen die (fach-)spezifischen Risiken und sind in der Lage, aktuell und angemessen darüber zu berichten. Angesichts der besonderen Zwecksetzung des kommunalen Risikomanagements besteht die Notwendigkeit, Risikomanagement auf Fachebene weiter zu professionalisieren und als wichtigen Baustein eines verwaltungsweiten Steuerungskonzeptes zu etablieren.

Allerdings ist darauf $\mathrm{zu}$ achten, möglichst keine isolierten „Insellösungen“ $\mathrm{zu}$ entwickeln, sondern Risiken möglichst verwaltungsweit abzustimmen und wechselseitige Abhängigkeiten auch ämterübergreifend zu erfassen.

7 Vgl. Schütz 2009, S. 122.

8 Vgl. Stadt Karlsruhe 2014, S. 2.

$9 \S 28$ Abs. 5 KomHVO NRW verweist auf die „Grundsätze zur ordnungsmäßigen Führung und Aufbewahrung von Büchern, Aufzeichnungen und Unterlagen in elektronischer Form sowie zum Datenzugriff“ (GoBD). Diese sehen die Einrichtung eines Internen Kontrollsystems (IKS) zwingend vor.

10 Vgl. KGSt 2011. 


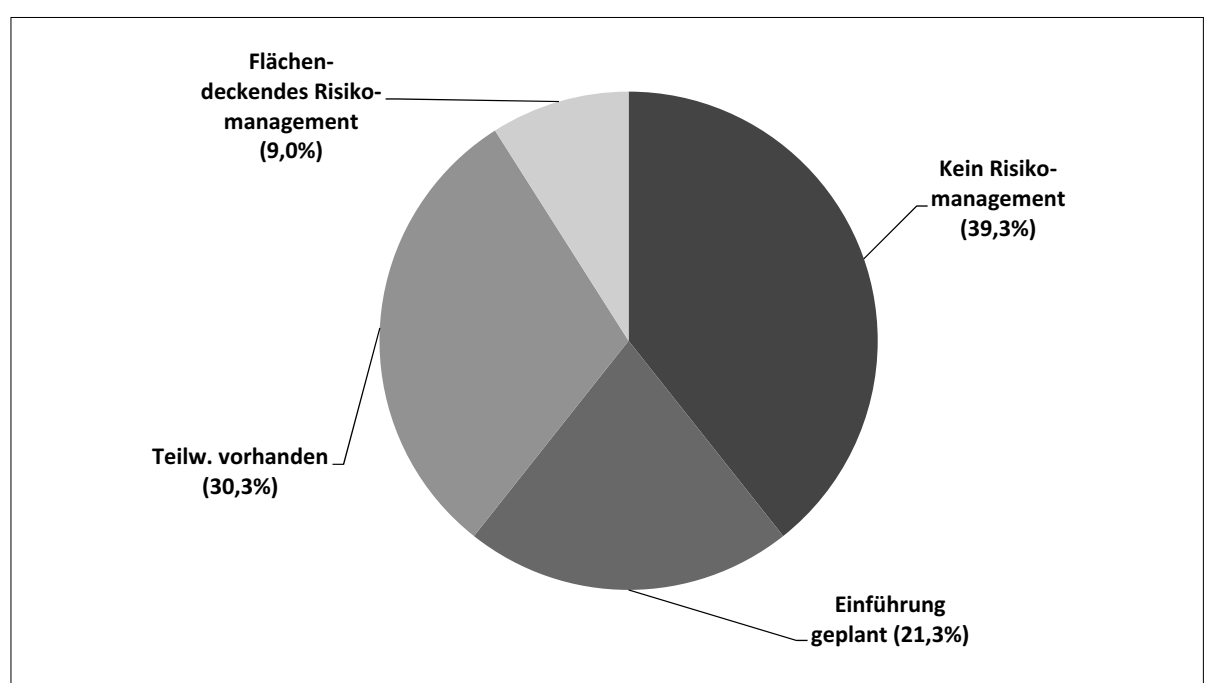

Abb. 3: Umsetzungsstand des Risikomanagements in Kommunen ( $n=122)$

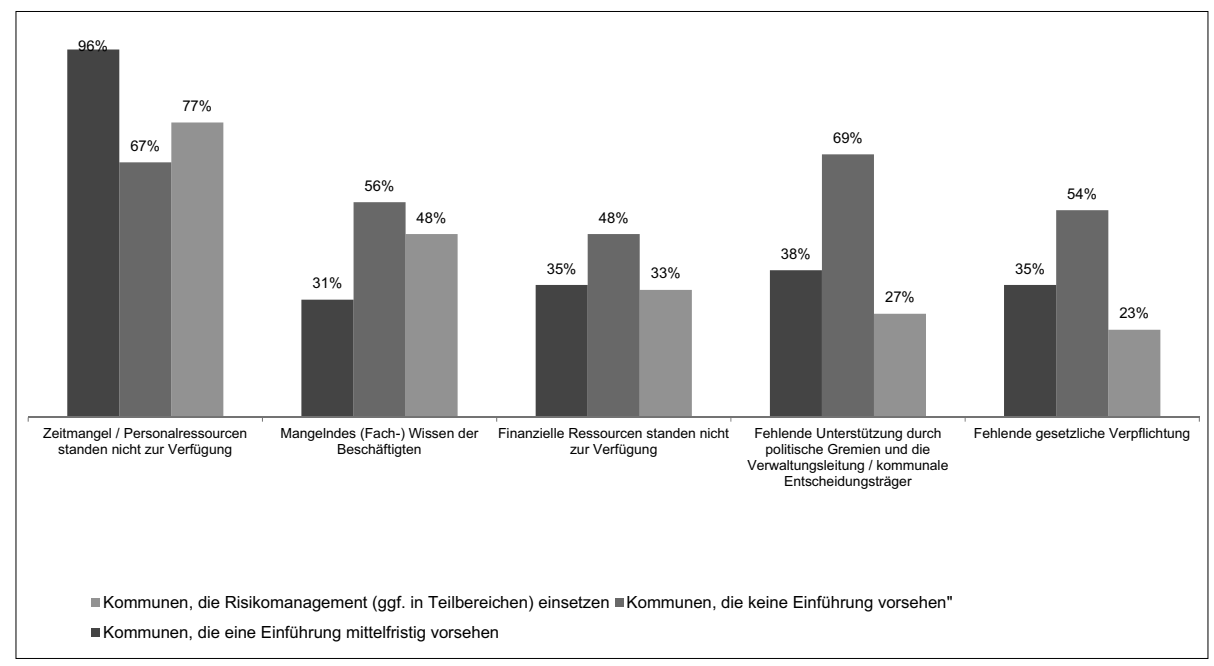

Abb. 4: Schwierigkeiten und Hindernisse bei der Einführung des kommunalen Risikomanagements

\section{Umsetzungsstand des kommuna- len Risikomanagements}

Obwohl mehr als die Hälfte der Befragten Risikomanagement für unverzichtbar hält, um die Verwaltung zu steuern, verfügen nur neun Prozent der Umfrageteilnehmer/-innen über ein flächendeckendes Risikomanagement. Knapp ein Drittel der Befragten gab an, zumindest in ausgewählten Teilbereichen über ein systematisches Risikomanagement zu verfügen. In jeder fünften Kommune ist die Einführung mittelfristig, d.h. innerhalb der nächsten fünf Jahre, vorgesehen (vgl. Abb. 3).

Die Mehrheit der Befragten hat Risikomanagement im Bereich Finanzmanagement/Kasse eingeführt. Der Befund, dass Kommunen der Steuerung von finanziellen Risiken einen hohen Stellenwert bei- messen, wird hierdurch nochmals unterstrichen.

Bezogen auf die Größenklassen kann festgestellt werden, dass größere Gemeinden und Landkreise eher über ein systematisches und dokumentiertes $\mathrm{Ri}$ sikomanagement verfügen als kleinere Kommunen. Geographisch betrachtet wurde Risikomanagement am häufigsten in ausgewählten (Teil-)Bereichen oder sogar verwaltungsweit in Bayern eingeführt $(63 \%)$, gefolgt von Nordrhein-Westfalen $(53 \%)$ und Niedersachsen $(45 \%)$. In den Bundesländern Sachsen, Schleswig-Holstein, Baden-Württemberg und Hessen wurde überwiegend noch kein Risikomanagement implementiert bzw. ist eine Einführung auch nicht vorgesehen. ${ }^{11}$ Dieses Resultat ist nur teilweise auf gesetzliche Gegebenheiten zurückzuführen. Bayern schreibt als einziges Bundesland in der
Gemeindeordnung vor, dass finanzielle Risiken bei der Führung der Haushaltswirtschaft zu minimieren sind (Art. 61 Abs. 3 GO). In Bezug auf den Lage- bzw. Rechenschaftsbericht ist nahezu bundesweit einheitlich geregelt, dass dort u.a. die zu erwartenden möglichen Risiken von besonderer Bedeutung darzustellen sind. ${ }^{12}$

Schwierigkeiten und Hindernisse, die der Einführung eines systematischen Risikomanagements entgegenstehen können, sind vor allem Zeitmangel, begrenzte Personalressourcen und die fehlende Unterstützung durch kommunale Entscheidungsträger. Unzureichendes (Fach-)Wissen der Beschäftigten, begrenzte finanzielle Mittel und fehlende rechtliche Vorgaben sind weitere Hemmnisse.

Abbildung 4 zeigt die Bedeutsamkeit der jeweiligen Hindernisse getrennt nach dem Reifegrad des Risikomanagements.

\section{Angemessene Fehler- und Risikokultur entwickeln}

Risikomanagement lässt sich nicht erfolgreich implementieren, wenn die Neigung besteht, Risiken zu verdrängen, anstatt offen mit ihnen umzugehen und rechtzeitig Gegensteuerungsmaßnahmen einzuleiten. Dies erfordert eine Führungs- und Organisationskultur, in der es allen Beschäftigten möglich und erlaubt ist, Risiken offen $\mathrm{zu}$ kommunizieren und transparent mit ihnen umzugehen. Häufig wird bereits der Begriff „Risiko“ missverstanden und die Existenz eines Risikos als persönlicher „Fehler“ interpretiert.

In jeder zweiten Kommune wird nach Ansicht der Befragten verwaltungsintern nicht angemessen über Risiken berichtet und ein bewusster Umgang mit Risiken seitens der Entscheidungsträger nicht gefördert. Auch waren 44 Prozent der Auffassung, dass Risiken nicht bewusst wahrgenommen und bei der Entscheidungsfindung berücksichtigt werden (vgl. Abb. 5).

11 Berücksichtigt wurden nur Bundesländer mit mehr als fünf vollständig ausgefüllten Fragebögen.

12 Zum Vergleich der einzelnen Regelungen siehe http://doppikvergleich.de/doppik/rechtsvergleich.html (Zugriff 22.02.2019). 
Die Einschätzung, dass verwaltungsintern nicht angemessen über Risiken berichtet wird, spiegelt sich auch in der externen Risikoberichterstattung wider. 44 Prozent der Umfrageteilnehmer/-innen halten die im Rechenschafts- bzw. Lagebericht aufgeführten Risikoinformationen für weniger relevant und aussagekräftig. ${ }^{13}$

Die bewusste Auseinandersetzung mit Chancen und Risiken sorgt für Transparenz und ist Grundlage einer verantwortungsvollen Verwaltungssteuerung. Die Entwicklung einer angemessenen - und auch von den kommunalen Entscheidungsträgern - ,gelebten “ Fehler- und Risikokultur („Tone at the Top“) ist ein entscheidender Faktor für die erfolgreiche Einführung und Nutzung eines systematischen Risikomanagements.

Politik und Verwaltungsleitung sind aufgefordert, den strategischen Rahmen und die Grundsätze für das Risikomanagement zu schaffen. Dies ist wesentliche Voraussetzung für die Akzeptanz bei jedem einzelnen Mitarbeitenden und den Mandatsträgern in der Kommune.

\section{Fehleranfälligkeit von Prozessen reduzieren}

Was den eigenen Arbeitsbereich bzw. das unmittelbare Arbeitsumfeld angeht, sind die Umfrageteilnehmer/-innen der Auffassung, dass mit Risiken effizient und wirkungsvoll umgegangen wird. Diese Einschätzung mag ein Grund dafür sein, warum Risikomanagement in vielen Kommunen noch nicht grundsätzlich systematisiert wird.

In jeder fünften Kommune treten jedoch häufig vermeidbare Fehler in wesentlichen Arbeitsprozessen auf. Bei zwei von drei Kommunen wurden in der Vergangenheit dolose Handlungen innerhalb der Gesamtverwaltung aufgedeckt. ${ }^{14}$

Die Fehleranfälligkeit von Prozessen spricht für eine systematische, risikoorientierte Überprüfung der wesentlichen Verwaltungsprozesse, den Aufbau eines Internen Kontrollsystems (IKS) ${ }^{15}$ und die Einrichtung und regelmäßige Überprüfung von Kontrollaktivitäten. Dies dient auch der Prävention von dolosen Handlungen.

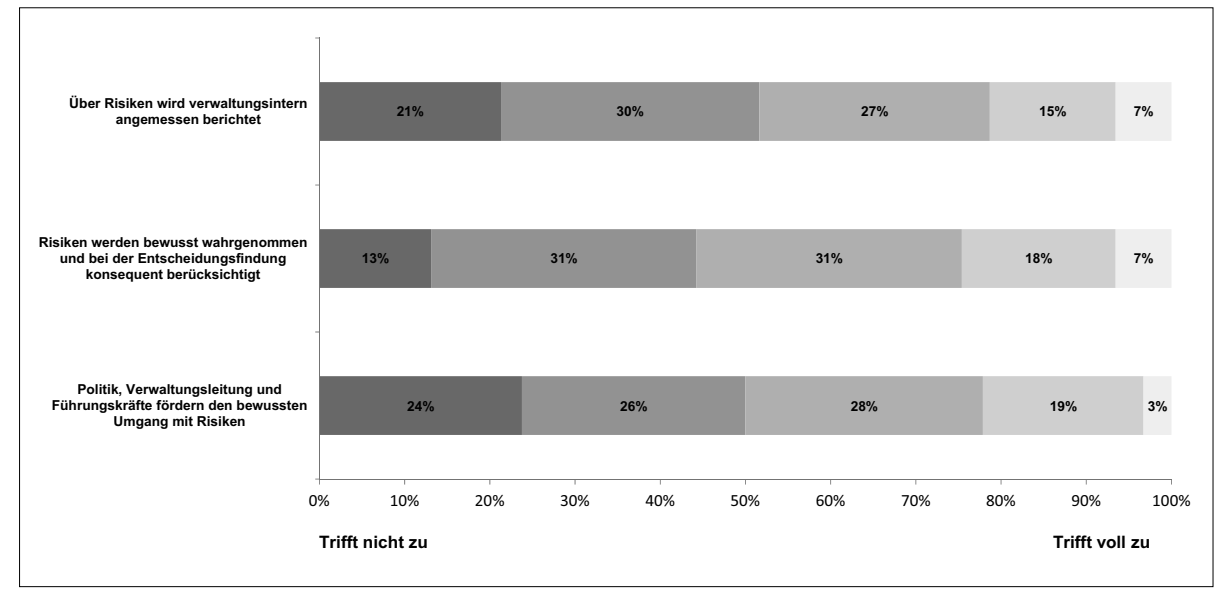

Abb. 5: Aussagen zur Risikoberichterstattung und zum Umgang mit Risiken $(n=122)$

In den meisten Kommunen führt das Auftreten von unerwünschten Ereignissen erst im Nachhinein zur Einleitung von Steuerungs- und Sanktionsmaßnahmen mit dem Ziel, Fehlentwicklungen und aufgetretene Schäden in Zukunft zu vermeiden. Der Schritt, Risikomanagement bereits im Vorfeld als wirkungsvolles Steuerungsinstrument zu nutzen und potenziellen Schäden damit frühzeitig und proaktiv entgegenzuwirken, steht in den meisten Kommunen allerdings noch aus.

\section{Pragmatischen Ansatz für das Risikomanagement wählen}

Neben der unzureichenden Unterstützung durch politische Gremien und die Verwaltungsleitung sind Zeitmangel und fehlende Personalressourcen ein entscheidendes Hindernis.

Es besteht daher die Notwendigkeit pragmatischer und weniger komplexer Ansätzen für ein kommunales Risikomanagement. Kommunen sollten einen Ansatz wählen, der der Größe der Verwaltung und dem örtlichen Steuerungsbedarf entspricht. Eine instrumentelle, organisatorische und personelle Anbindung an bestehende Planungs-, Steuerungs- und Kontrollsysteme ist ein geeigneter Lösungsansatz, um personelle und zeitliche Engpässe zu überwinden und Kontinuität

13 Zur Risikoberichterstattung im Lagebericht vgl. auch Burth/Hilgers 2012.

14 Diebstahl, Betrug und Untreue, Unterschlagung, Urkundenfälschung, Korruption u.ä. werden als dolose Handlungen (nach lateinisch dolosus = arglistig, trügerisch) bezeichnet.

15 Vgl. KGSt 2014; Hunziker et al. 2015. sicher zu stellen. Risikomanagement ist in diesem Fall keine eigenständige Aufgabe, sondern integraler Bestandteil des verwaltungsweiten Steuerungskonzeptes. So ergeben sich insbesondere Schnittstellen und Schnittmengen zwischen Controlling, Risikomanagement und dem IKS, das dazu beiträgt, Prozessrisiken mittels wirksamer Kontrollaktivitäten frühzeitig zu erkennen und bei Bedarf entsprechende Gegensteuerungsmaßnahmen einzuleiten. Durch ergänzende Risikoinformationen wird die Aussagekraft von Controlling-Berichten weiter verbessert. ${ }^{16}$

Die Bereitstellung von Risikoinformationen muss zudem adressatengerecht erfolgen und in einem angemessenen Verhältnis zum Steuerungsnutzen stehen. Insofern empfiehlt sich eine Konzentration auf wesentliche Risiken. Ein weit verbreiteter Ansatz zur Risikobewertung und anschließenden Risiko Priorisierung im Sinne einer Kommunikationshilfe sind Risikoportfolios bzw. -landkarten („Risk Maps“). ${ }^{17}$

\section{Fach- und Methodenkompetenz vermitteln}

Risikoidentifikation, -bewertung und -steuerung erfordern neue Kenntnisse und Fähigkeiten der betroffenen Mitarbeiterinnen und Mitarbeiter. Werden diese Fä-
16 Zur Vernetzung von Controlling und Risikomanagement vgl. Risk Management Association e.V./Internationaler Controller Verein e.V. 2018; Klein/Gleißner 2017 sowie Vanini 2012, S. 263 ff. Bezogen auf die Unternehmensplanung und Budgetierung auch Gräf/Höhner 2004.

17 Vgl. Hunziker/Meissner 2017, S. $42 \mathrm{ff}$. 
higkeiten und Kenntnisse nicht gezielt und adressatengerecht aufgebaut, so sind fehlende Akzeptanz des Risikomanagements und möglicherweise sogar Ablehnung die Folgen.

Daher ist es wichtig, dass das Wissen zum Risikomanagement breit verankert ist und nicht nur von einzelnen Personen vorangetrieben wird. Schulungen, Workshops, der interkommunale Erfahrungsaustausch und Grundsatzerklärungen der Verwaltungsleitung zum Umgang mit Risiken tragen dazu bei, eine angemessene Risiko- und Fehlerkultur aufzubauen und den Beschäftigten die nötige Fach- und Methodenkompetenz zu vermitteln.

Ebenso können technikunterstützte Tools und geeignete Softwarelösungen unterschiedlicher Priorität betrieben wird, welche Schwierigkeiten und Hindernisse sich bei der Einführung ergeben und welche Rahmenbedingungen ein erfolgreiches Risikomanagement begünstigen.

Viele Kommunen haben Risikomanagement (zunächst) im Finanzbereich implementiert bzw. die Einführung innerhalb der nächsten fünf Jahre vorgesehen. Allerdings wird auch der Abwehr von Sach- und Vermögensschäden, von Gefahren für Mensch und Umwelt, der Einhaltung von rechtlichen Vorgaben sowie der Korruptionsvorbeugung und Vermeidung von Reputationsschäden ein hoher Stellenwert beigemessen. Damit umfasst das kommunale Risikomanagement nicht nur die Abwehr finanzieller Risiken. Betroffen ist auch die Fachebene mit dem Ziel,

\section{"Erfolgreiches Risikomanagement zeichnet sich dadurch aus, dass offen über Risiken kommuniziert und transparent mit ihnen umgegangen wird. Durch eine offene Risikokultur lassen sich potenzielle Risiken im Vorfeld erkennen und Fehler in wesentlichen Arbeitsprozessen vermeiden."}

siken kommuniziert und transparent mit ihnen umgegangen wird. Ein Risiko darf nicht als persönlicher Fehler missverstanden werden. Durch eine offene Risikokultur lassen sich potenzielle Risiken im Vorfeld erkennen und Fehler in wesentlichen Arbeitsprozessen vermeiden.

Bei der Einführung sollten Kommunen einen pragmatischen Ansatz entsprechend der Gemeindegröße und -komplexität wählen. Vor allem in kleineren Kommunen ist eine organisatorische Verselbstständigung des Risikomanagements oftmals nicht möglich (und auch nicht nötig oder sinnvoll). Es empfiehlt sich, Schnittstellen und Schnittmengen zu identifizieren und zu prüfen, wo eine Vernetzung vorhandener Steuerungsinstrumente möglich ist. Dies ist ein geeigneter Lösungsansatz, um Zeitmangel und begrenzten Personalressourcen zu begegnen.

Schließlich muss ein erfolgreiches Risikomanagement sicherstellen, dass die Mitarbeiterinnen und Mitarbeiter über die nötige Fach- und Methodenkompetenz zum Umgang mit Risiken verfügen. Schulungen und ein regelmäßiger Austausch tragen dazu bei, die erforderlichen Kompetenzen innerhalb der Verwaltung aufzubauen und zu verbreiten. Zur Dokumentation und Kommunikation von Risiken kann auch der Einsatz von technischen Hilfsmitteln und geeigneten Softwarelösungen hilfreich sein.
(Microsoft Excel, Spezialsoftware von Drittanbietern) Kommunen bei der systematischen Dokumentation und Bewertung von Risiken unterstützen. Dies setzt allerdings voraus, dass die notwendigen (finanziellen) Ressourcen für die Beschaffung und den laufenden Betrieb zu Verfügung stehen.

\section{Fazit}

Ein systematischer Umgang mit Risiken gewinnt in Kommunen zunehmend an Bedeutung. Allerdings steht die Herausforderung, dieses Managementinstrument stärker in der Praxis zu verankern, in vielen Kommunen noch aus. Ziel der Umfrage war es, einen Überblick über den Stand des Risikomanagements zu gewinnen. Die Umfrage sollte Anhaltspunkte liefern, weshalb Risikomanagement vor Ort mit
Risiken gemeindeweit zu steuern und die Zielerreichung im Kontext des vielschichtigen kommunalen Leistungsspektrums sicherzustellen. Dabei darf nicht vergessen werden, dass Risiken auf Fachebene meist mit finanziellen Risiken einhergehen.

Bei der Einführung gilt es, unterschiedlichen Herausforderungen $\mathrm{zu}$ begegnen. Hierzu zählen insbesondere Zeitmangel und begrenzte Personalressourcen. Auch die fehlende Unterstützung durch kommunale Entscheidungsträger trägt dazu bei, dass ein systematisches kommunales Risikomanagement noch nicht weit verbreitet ist. Politik und Verwaltungsleitung sind gefordert, den strategischen Rahmen zu setzen.

Erfolgreiches Risikomanagement zeichnet sich dadurch aus, dass offen über $\mathrm{Ri}$ - 


\section{Literatur}

Blättler, S./Hunziker, S. (2015): Kommunales Risikomanagement: Erfahrungen und Empfehlungen, in: Verwaltung \& Management, Heft 2/2015, S. 83-88.

Budäus, D./Hilgers, D. (2009): Öffentliches Risikomanagement - zukünftige Herausforderungen an Staat und Verwaltung, in: Scholz, F./ Schuler, A./Schwintowski, H.-P. (Hrsg.): Risikomanagement der öffentlichen Hand, Heidelberg, S. 17-78.

Burth, A./Hilgers, D. (2012): Kommunale Risikoberichterstattung. Eine vergleichende Analyse doppischer Lageberichte, in: Verwaltung \& Management, Heft 1/2012, S. 7-16.

Gräf, J./Höhner, M. (2004): Integration von Risikomanagement in die Unternehmensplanung und in die Budgetierung, in: Horváth \& Partners (Hrsg.): Beyond Budgeting umsetzen. Erfolgreich planen mit Advanced Budgeting, Stuttgart, S. 195-205.

Hunziker, S./Dietiker, Y./Schiltz, K./Gwerder, L. (2015): Ganzheitliche Risikosteuerung in 10 Schritten. Risikomanagement und IKS für Schweizer Gemeinden, Bern.

Hunziker, S./Meissner, J. (2017): Risikomanagement in 10 Schritten, Wiesbaden.

Kommunale Gemeinschaftsstelle für Verwaltungsmanagement (KGSt) (2019): Umsetzungsstand des kommunalen Risikomanagements. Ergebnisse einer Umfrage: Schlussfolgerungen und Handlungsempfehlungen, Bericht 1/2019.

Kommunale Gemeinschaftsstelle für Verwaltungsmanagement (KGSt) (2011): Kommunales Risikomanagement - Teil 1: Das kommunale Risikofrühwarnsystem, Bericht 5/2011.

Kommunale Gemeinschaftsstelle für Verwaltungsmanagement (KGSt) (2014): Kommunales Risikomanagement - Teil 2: Das interne Kontrollsystem, Bericht 8/2014.

Klein, A./Gleißner, W. (Hrsg.) (2017): Harmonisierung von Controlling und Risikomanagement, Freiburg.

Risk Management Association e.V./Internationaler Controller Verein e.V. (Hrsg.) (2018): Vernetzung von Risikomanagement und Controlling. Grundlagen - Praktische Aspekte Synergiepotenziale. Berlin.

Schütz, M. (2009): Risikomanagement aus Sicht des Öffentlichen Sektors der Schweiz, in: Scholz, F./Schuler, A./Schwintowski, H.-P. (Hrsg.): Risikomanagement der öffentlichen Hand, Heidelberg, S. 117-154.

Stadt Karlsruhe (2014): Erstinformation Risikomanagement, unveröffentlichtes Dokument.

Vanini, U. (2012). Risikomanagement. Grundlagen - Instrumente - Unternehmenspraxis, Stuttgart.

\title{
Der Bundesrat in der Parteiendemokratie
}

\author{
Das Zustandekommen von Entscheidungen \\ der Länderkammer
}

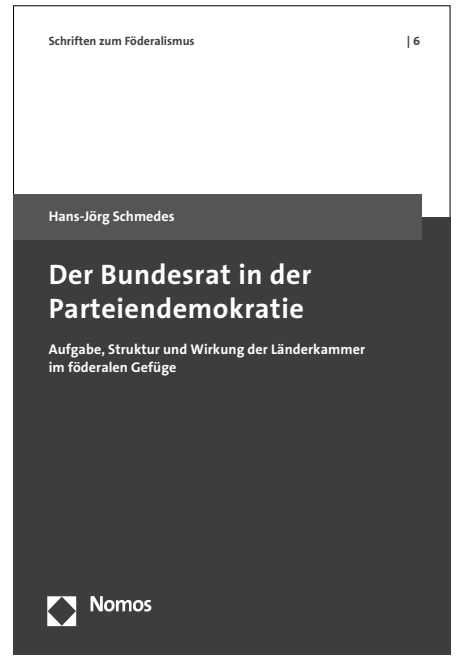

In Deutschland wird das Spannungsverhältnis zwischen parlamentarischer Konkurrenz- und bundesstaatlicher Verhandlungsdemokratie in besonderer Weise durch den Bundesrat verkörpert, dessen Strukturen und Verfahren bei der Koordination von Interessen des Bundes und der Länder in der deutschen Politikverflechtung allerdings als undurchsichtig und wenig nachvollziehbar gelten. In der öffentlichen Diskussion befördert eine fehlende parteipolitische Mehrheit einer Bundesregierung im Bundesrat häufig zudem die Sorge vor parteitaktisch motivierten Blockadehaltungen der Länderkammer. Tatsächlich sind die Entscheidungsfindungsprozesse der Länderkammer durch ein Ineinandergreifen von formell-administrativen und informell-politischen Koordinationsstrukturen gekennzeichnet, die von den

\section{Der Bundesrat in der Parteiendemokratie}

Aufgabe, Struktur und Wirkung der Länderkammer im föderalen Gefüge Von Prof. Dr. Hans-Jörg Schmedes 2019, 116 S., brosch., 24,- $€$ ISBN 978-3-8487-5509-7 (Schriften zum Föderalismus, Bd. 6) nomos-shop.de/40617

Strukturen und Gesetzmäßigkeiten der in ihr wirkenden Parteien überlagert werden.

Der Autor untersucht Rolle, Tätigkeit und Verfahren des Bundesrates und stellt den Ablauf der administrativen und (partei-)politischen Koordination vor. Zudem wird dargelegt, dass in der 17. und 18. Wahlperiodetrotz teilweise gegenläufiger Mehrheiten von Bundestag und Bundesrat nur äußerst wenige Gesetze an einer Zustimmungsversagung der Länderkammer abschließend gescheitert sind, so dass man (auch) für diesen Zeitraum von einer konkordanzdemokratisch geprägten Phase des deutschen Regierungssystems sprechen kann.

Der Autor: Hans-Jörg Schmedes ist Honorarprofessor an der HumboldtUniversität zu Berlin und Referatsleiter in der Senatskanzlei des Landes Berlin.
હิ

Bestellen Sie jetzt telefonisch unter (+49)7221/2104-37. Portofreie Buch-Bestellungen unter www.nomos-shop.de Alle Preise inkl. Mehrwertsteuer 